\title{
Sex Differences in the Gut Microbiota as Potential Determinants of Gender Predisposition to Disease
}

\author{
Jose A. Santos-Marcos, Carmen Haro, Ana Vega-Rojas, Juan F. Alcala-Diaz, Helena \\ Molina-Abril, Ana Leon-Acuña, Javier Lopez-Moreno, Blanca B. Landa, Manuel Tena- \\ Sempere, Pablo Perez-Martinez, Jose Lopez-Miranda, \\ Francisco Perez-Jimenez, and Antonio Camargo*
}

Scope: Dysbiosis of gut microbiota is involved in metabolic syndrome (MetS) development, which has a different incidence between men $(M)$ and women (W). The differences in gut microbiota in MetS patients are explored according to gender, and whether consuming two healthy diets, Mediterranean (MED) and low-fat (LF), may, over time, differentially shape the gut microbiota dysbiosis according to gender is evaluated.

Materials and Methods: All the women from the CORDIOPREV study whose feces samples were available and a similar number of men, matched by the main metabolic variables ( $N=246,123$ women and 123 men), and categorized according to the presence or not of MetS are included. Gut microbiota is analyzed at baseline and after $\mathbf{3}$ years of dietary intervention. Results: Higher abundance of Collinsella, Alistipes, Anaerotruncus, and Phascolarctobacterium genera is observed in MetS-W than in MetS-M, whereas the abundance of Faecalibacterium and Prevotella genera is higher in MetS-M than in MetS-W. Moreover, higher levels of Desulfovibrio, Roseburia, and Holdemania are observed in men than in women after the consumption of the LF diet.

Conclusion: The results suggest the potential involvement of differences in gut microbiota in the unequal incidence of metabolic diseases between genders, and a sex-dependent effect on shaping the gut microbiota according to diet.

\section{Introduction}

The incidence of metabolic diseases and their comorbidities are sexually dimorphic. ${ }^{[1]}$ For example, the prevalence of MetS, a cluster of characteristics associated with an increased risk of type 2 diabetes mellitus (T2DM) and cardiovascular diseases, ${ }^{[2]}$ differs by age, ethnicity, gender, diet, and levels of physical activity. ${ }^{[3]}$ This syndrome is conventionally diagnosed when a threshold of three of five criteria is reached; yet, ten different combinations of such criteria are in fact possible, each with a different pathophysiology. Moreover, the prevalence of each metabolic syndrome risk factor also differs by gender and country. ${ }^{[4,5]}$

Certain fundamental aspects of metabolic homeostasis are regulated differently in males and females. ${ }^{[6]}$ For example, women have a propensity to store fat in subcutaneous adipose tissue compared to the preferential visceral fat deposition in men. ${ }^{[7]}$ However, females seem to be more predisposed to obesity and metabolic syndrome than men, ${ }^{[8]}$
J. A. Santos-Marcos, C. Haro, A. Vega-Rojas, Dr. J. F. Alcala-Diaz, Dr. A. Leon-Acuña, Dr. J. Lopez-Moreno, Prof. P. Perez-Martinez, Prof. J. Lopez-Miranda, Prof. F. Perez-Jimenez, A. Camargo Lipids and Atherosclerosis Research Unit

Maimonides Biomedical Research Institute of Cordoba (IMIBIC), Reina Sofia University Hospital, University of Cordoba

Cordoba 14004, Spain

E-mail: antonio.camargo@imibic.org

J. A. Santos-Marcos, C. Haro, A. Vega-Rojas, Dr. J. F. Alcala-Diaz, Dr. A. Leon-Acuña, Dr. J. Lopez-Moreno, Prof. M. Tena-Sempere, Prof. P. Perez-Martinez, Prof. J. Lopez-Miranda, Prof. F. Perez-Jimenez, A. Camargo

CIBER Fisiopatologia de la Obesidad y Nutricion (CIBEROBN) Instituto de Salud Carlos III

Cordoba 14004, Spain

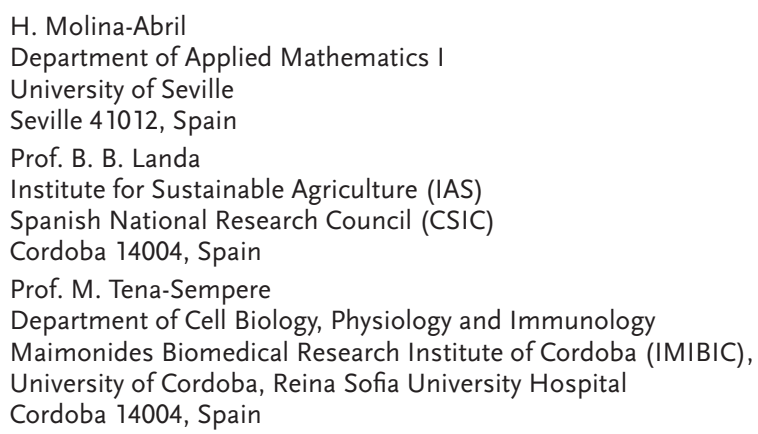


despite the fact that visceral fat has a stronger association with cardiovascular and metabolic disease. ${ }^{[9]}$ It has also been described that glucose homeostasis is subject to sexual asymmetry. In fact, the prevalence of prediabetic syndromes differs by genders. Whereas impaired fasting glucose (IFG) is more prevalent in men, impaired glucose tolerance (IGT) is more prevalent in women. ${ }^{[6]}$ In addition, there are more diabetic males before puberty and more diabetic females after menopause. ${ }^{[10]}$

Gut microbiota is now recognized as an organ integrated in the metabolism of the host. ${ }^{[11]}$ It has been proposed that alteration of the gut microbiota structure may trigger the development of metabolic diseases such as obesity, MetS, and T2DM. ${ }^{[12-14]}$ Taking into account that previous data indicate that the composition of the gut microbiota is different between genders, ${ }^{[15-18]}$ we hypothesized that the microbiota gender difference might determine the differences in the predisposition to develop MetS between women and men.

Dietary strategies are of great interest in preventing and treating metabolic diseases ${ }^{[19]}$ and it has been suggested that they act by modifying the microbiome. ${ }^{[20]}$ Although it has been suggested that the composition of the gut microbiota in adulthood is very stable over time, ${ }^{[21]}$ the consumption of different diets can shape the gut microbiota and could potentially be used to restore an imbalanced microbiome to a healthy, balanced microbiome. ${ }^{[20]}$ However, the effectiveness of diets may be dependent on microbiota composition of the recipient, as has been shown in the case of fecal transplant response, ${ }^{[22]}$ suggesting that a different gut microbiota composition in men and women before dietary intervention may be a determinant in the effect of diet in modifying the microbiome between genders.

Based on this previous evidence, we explored the gut microbiota composition in men and women with or without MetS and also evaluated if the consumption of Mediterranean (MED) and low-fat (LF) diets for 3 years may differentially shape the gut microbiota composition according to gender.

\section{Experimental Section}

\subsection{Study Participants}

The current work was conducted in the framework of the CORDIOPREV study (Clinical Trials.gov.Identifier: NCT00924937), an ongoing prospective, randomized, open, controlled trial in 1002 patients with coronary heart disease (CHD) who had their last coronary event over 6 months before enrolling. The patients, in addition to conventional treatment for CHD, followed MED and LF diets over a period of 7 years. The inclusion and exclusion criteria have been previously described, ${ }^{[23]}$ and are summarized thus: patients were eligible if they were over 20 years of age but below 75, had established CHD without clinical events in the last 6 months, were thought to be capable of following a long-term dietary intervention, and did not have severe diseases or an estimated life expectancy of below 7 years. For the current study, the use of antibiotics was added as one of the exclusion criteria. All the patients gave their informed consent in writing to participate in the study. The trial protocol and all the amendments were approved by the local ethics committees, following the Helsinki Declaration and good clinical practice.
The criteria of the Third Report of the National Cholesterol Education Program (NCEP) Expert Panel on Detection, Evaluation, and Treatment of High Blood Cholesterol in Adults (Adult Treatment Panel III) were followed to assess the presence of MetS. ${ }^{[2]}$ All the women from the CORDIOPREV study whose feces samples were available, and a similar number of men $(\mathrm{N}=246,123$ women and 123 men), were included in this study and categorized according to the presence or not of MetS as follows: MetSW, 79 women with MetS; MetS-M, 79 men with MetS; NonMetSW, 44 women without MetS; NonMetS-M, 44 men without MetS. Each woman was individually matched to one man according to the presence of MetS, T2DM (presence, treatment, and if treatment was with metformine), fasting glucose criteria for MetS, HbA1c levels (according to American Diabetes Association criteria for normoglucemia, prediabetes, and T2DM), triacylglycerides (TAG), HDL-c, hypertension criteria for MetS and age (ranges: <40, 40-49, 50-59, 60-69, and >70). This matching was performed in order to avoid differences between genders in variables that may affect gut microbiota composition different to the MetS. In fact, the women and men shared the same combinations of MetS criteria (Table S1, Supporting Information). In addition, we checked the nutritional background between genders and groups of patients according the presence of MetS (Table S2, Supporting Information), which may also affect gut microbiota composition. Moreover, there were no differences on physical activity between groups.

The baseline metabolic characteristics of the patients in the study are shown in Table 1. The effect of the consumption of MED or LF diets for 3 years in shaping the gut microbiota of the MetS patients was performed in a smaller population (99 of the 158 MetS patients), due to the availability of fecal samples at 3 years of follow-up. The baseline metabolic characteristics and the presence of MetS criteria of the patients included in the diet effect analysis are shown in Table S5, Supporting Information.

\subsection{Diet Assessment}

The adherence to the MED diet was assessed by a validated 14item questionnaire ${ }^{[25]}$ and the adherence to the LF diet by a 9-point score. This was performed once before the start of the dietary intervention and then yearly. The Spanish food composition tables and a validated food frequency questionnaire ${ }^{[26]}$ were used to calculate the intake of fiber.

\subsection{Clinical Plasma Parameters}

Tubes containing $0.1 \%$ EDTA were used to collect the blood, which were then centrifuged at $1500 \times \mathrm{g}$ for $15 \mathrm{~min}$ at $4{ }^{\circ} \mathrm{C}$ to separate the plasma and red blood cells. The analytes were determined, blinded to the team members, from frozen samples at the Lipid and Atherosclerosis Unit at Reina Sofia University Hospital by members of the laboratory research team, as previously described. ${ }^{[17]}$

\subsection{DNA Extraction from Fecal Samples}

The patients were given a box with carbonic ice and a sterile plastic bottle with a screw cap to collect the fecal samples. The 
Table 1. Baseline characteristic of the participants in the study. Values correspond to the mean \pm SEM.

\begin{tabular}{|c|c|c|c|c|c|}
\hline & MetS-W $(N=79)$ & MetS-M $(N=79)$ & NonMetS-W $(N=44)$ & NonMetS-M $(N=44)$ & $p$-Value \\
\hline Age (years) & $62.95 \pm 1.00$ & $61.63 \pm 0.99$ & $61.48 \pm 1.27$ & $60.61 \pm 1.34$ & 0.526 \\
\hline BMI $\left(\mathrm{kg} \mathrm{m}^{-2}\right)$ & $32.18 \pm 0.46^{\mathrm{a}}$ & $31.83 \pm 0.51^{\mathrm{a}, \mathrm{c}}$ & $29.41 \pm 0.88^{b}$ & $29.88 \pm 0.61^{b, c}$ & 0.002 \\
\hline Waist circumference $(\mathrm{cm})$ & $102.37 \pm 1.19^{a}$ & $110.83 \pm 1.45^{b}$ & $93.29 \pm 2.13^{c}$ & $103.61 \pm 1.58^{\mathrm{a}}$ & $<0.001$ \\
\hline HDL-c $\left(\mathrm{mg} \mathrm{dL}^{-1}\right)$ & $42.56 \pm 1.21^{a}$ & $37.35 \pm 1.01^{b}$ & $57.30 \pm 1.63^{c}$ & $45.91 \pm 1.56^{\mathrm{a}}$ & $<0.001$ \\
\hline LDL-c $\left(\mathrm{mg} \mathrm{dL}^{-1}\right)$ & $88.48 \pm 3.34^{\mathrm{a}, \mathrm{b}}$ & $82.71 \pm 2.58^{\mathrm{a}}$ & $97.73 \pm 4.03^{b}$ & $94.30 \pm 4.33^{\mathrm{a}, \mathrm{b}}$ & 0.014 \\
\hline Total cholesterol $\left(\mathrm{mg} \mathrm{dL}^{-1}\right)$ & $164.46 \pm 4.53^{\mathrm{a}, \mathrm{b}}$ & $156.84 \pm 2.94^{\mathrm{a}}$ & $175.77 \pm 4.54^{\mathrm{b}}$ & $160.55 \pm 5.25^{\mathrm{a}, \mathrm{b}}$ & 0.026 \\
\hline TAG $\left(\mathrm{mg} \mathrm{dL}^{-1}\right)$ & $159.36 \pm 9.39^{\mathrm{a}}$ & $168.40 \pm 8.58^{\mathrm{a}}$ & $96.27 \pm 5.37^{\mathrm{b}}$ & $99.93 \pm 5.38^{b}$ & $<0.001$ \\
\hline Clucose $\left(\mathrm{mg} \mathrm{dL}^{-1}\right)$ & $130.13 \pm 6.50^{\mathrm{a}}$ & $126.49 \pm 4.68^{\mathrm{a}}$ & $90.07 \pm 1.90^{b}$ & $93.27 \pm 2.43^{b}$ & $<0.001$ \\
\hline Insulin $\left(m U L^{-1}\right)$ & $12.58 \pm 1.62^{\mathrm{a}, \mathrm{b}}$ & $14.48 \pm 1.66^{\mathrm{a}}$ & $7.01 \pm 0.62^{b}$ & $8.58 \pm 1.02^{\mathrm{a}, \mathrm{b}}$ & 0.003 \\
\hline HbAlc (\%) & $7.11 \pm 0.17^{\mathrm{a}}$ & $6.99 \pm 0.15^{\mathrm{a}}$ & $6.09 \pm 0.08^{b}$ & $6.27 \pm 0.11^{\mathrm{b}}$ & $<0.001$ \\
\hline Sistolic BP (mm Hg) & $145.06 \pm 2.22^{\mathrm{a}}$ & $146.60 \pm 2.23^{\mathrm{a}}$ & $133.70 \pm 3.19^{\mathrm{b}}$ & $127.84 \pm 2.58^{b}$ & $<0.001$ \\
\hline Diastolic BP (mm Hg) & $75.28 \pm 1.22^{\mathrm{a}}$ & $80.10 \pm 1.31^{b}$ & $72.86 \pm 1.36^{\mathrm{a}}$ & $70.07 \pm 1.75^{\mathrm{a}}$ & $<0.001$ \\
\hline
\end{tabular}

HDL-c, HDL-cholesterol; LDL-c, LDL-cholesterol; TAG, triacylglycerides; and BP, blood pressure. The statistical differences between groups were evaluated by one-way ANOVA. In each row, values with different letters in superscript differ statistically in the Bonferroni's post hoc test $(p<0.05)$.

samples were kept frozen after delivery to the laboratory staff and stored at $-80^{\circ} \mathrm{C}$. DNA was extracted using the QIAamp DNA kit Stool Mini Kit Handbook (Qiagen, Hilden, Germany), following the manufacturer's instructions. DNA samples were stored at $-20^{\circ} \mathrm{C}$, after quantification with the Nanodrop ND-1000 v3.5.2 spectrophotometer (Nanodrop Technology, Cambridge, UK).

\subsection{Sequencing and Bioinformatics}

For each DNA (fecal) sample, the hypervariable regions V3 and V4 of the 16S rRNA gene were amplified by polymerase chain reaction using the primer pair 5'-TCGTCGGCAGCGTCAGATG TGTATAAGAGACAG- $3^{\prime}$ and $5^{\prime}$-GTCTCGTGGGCTCGGAGATG TGTATAAGAGACAG-3', ${ }^{[27]}$ which was further sequenced on a MiSeq Illumina platform (Illumina, San Diego, CA). Briefly, PCR was performed using a KAPA HiFi HotStart ReadyMix (KAPABIOSYSTEMS), $1.25 \mu \mathrm{L}$ of extracted DNA $\left(5 \mathrm{ng} \mu \mathrm{L}^{-1}\right.$ in $10 \mathrm{~mm}$ Tris pH8.5) and $0.2 \mu \mathrm{M}$ of each primer, using the following cycle parameters: 3 min denaturation at $95{ }^{\circ} \mathrm{C}$ followed by 25 cycles $\left(30 \mathrm{~s}\right.$ at $95^{\circ} \mathrm{C}, 30 \mathrm{~s}$ at $60{ }^{\circ} \mathrm{C}, 30 \mathrm{~s}$ at $72{ }^{\circ} \mathrm{C}$ ) and a final extension at $72{ }^{\circ} \mathrm{C}$ for $5 \mathrm{~min}$. The $16 \mathrm{~S} \mathrm{~V} 3$ and V4 amplicon purification was performed using Agentcourt AMPure XP beads (Beckman Coulter). A second PCR reaction attaches dual indices and Illumina sequencing adapters using the Nextera XT Index Kit. This PCR was performed with a KAPA HiFi HotStart ReadyMix (KAPABIOSYSTEMS), $5 \mu \mathrm{L}$ of the previous amplicon, $5 \mu \mathrm{L}$ of each Nextera XT Index Primer 1(N7xx), and $5 \mu \mathrm{L}$ of each Nextera XT Index Primer 2(S5xx), using the following cycle parameters: 3 min denaturation at $95^{\circ} \mathrm{C}$ followed by 8 cycles $\left(30 \mathrm{~s}\right.$ at $95^{\circ} \mathrm{C}, 30 \mathrm{~s}$ at $55^{\circ} \mathrm{C}, 30 \mathrm{~s}$ at $72{ }^{\circ} \mathrm{C}$ ), and a final extension at $72{ }^{\circ} \mathrm{C}$ for $5 \mathrm{~min}$. The PCR product purification was performed using Agentcourt AMPure XP beads (Beckman Coulter). Sequence outputs were analyzed using the Quantitative Insights into Microbial Ecology (QIIME) program, version 1.9.1, ${ }^{[28]}$ using QIIME default parameters. The $16 \mathrm{~S}$ paired reads were assembled using the script multiple_join_paired_ends.py, which joins forward and reverse demultiplexed reads. The output file was processed for quality filtering by split_libraries_fastq.py. High quality sequences were grouped into Operational Taxonomic Units (OTUs) with a sequence identity threshold of $97 \%$, and taxonomy was assigned by interrogating the high quality sequences with the Greengenes database (13_5). ${ }^{[29]}$ Bacterial richness and diversity across the samples were calculated using the Chao1, Simpson, and Shannon indexes. ${ }^{[30]}$ Principal component analysis of community structure (beta-diversity) was done using the unweighted and weighted UniFrac distance metrics ${ }^{[31]}$ and analyzed by permutational multivariate analysis of variance (PERMANOVA) using the script compare_categories.py. Linear discriminant analysis (LDA) effect size (LEfSe) (http://huttenhower.sph.harvard.edu/galaxy/) was used to compare groups at baseline and visualize the results using taxonomic bar charts and cladograms. ${ }^{[32]}$

\subsection{Statistical Analysis}

R software, version 3.0.2 (R Foundation for Statistical Computing, http://www.R-project.org/) was used for men and women matching with the MatchIt 3.0.2. PASW statistical software package, version 20.0 (IBM Inc., Chicago, IL, USA), which was used for univariate statistical analyses of the data. The statistical differences in the abundance of bacterial groups at baseline were tested by one-way analysis of variance (ANOVA). This test was adjusted by the false discovery rate (FDR) using the Benjamini and Hochberg method. Gut microbiota changes according to the dietary intervention were assessed by ANOVA for repeated measures, with time as intrasubject factor, and diet and gender as the intersubject factors. Post hoc statistical analysis was completed using Bonferroni's comparison test. To evaluate the specific differences between bacterial taxa, the abundance of taxa present in at least $75 \%$ of the human fecal DNA samples in each of the study groups was compared. Moreover, the frequency of occurrence of the bacterial taxa identified in at least $50 \%$ of the samples was analyzed in each of the groups in the study using the $\chi^{2}$ test. $p$-Values $<0.05$ and $q$-values $<0.1$ were considered statistically significant in all the statistical analyses. All the data shown in this study have been expressed as mean \pm SEM. 


\section{Results}

\subsection{Baseline Characteristics of the Study Participants}

The differences in the main anthropometric and metabolic variables between groups are shown in Table 1. Overall, both MetS groups of women and men had higher waist circumference, glucose, TAG, and systolic blood pressure, and lower HDL-c plasma levels than NonMetS groups (women and men). The MetS criteria were similar between genders (Table S1, Supporting Information). In addition, the nutritional background was similar between genders, and between MetS and NonMetS groups (Table S2, Supporting Information).

\subsection{Relationship between Anthropometric and Metabolic Variables with Gut Microbiota Composition}

We studied the relationship between the main anthropometric and metabolic variables at baseline and the gut microbiota composition using Pearson's correlation test. We found a positive relationship between waist circumference and the Acidaminococcus genus $(R=0.592, p<0.001)$ in men with MetS, and a negative relationship between c-HDL plasma concentration and the Desulfovibrio genus $(R=-0.568, p<0.001)$ in men without MetS. Moreover, we also observed positive relationships between $\mathrm{HbA} 1 \mathrm{C}(\%)$ and an unknown genus from the S24-4 family ( $R=0.514, p=0.001$ ), the plasma concentration of TAG and an unknown genus from Ruminococcaceae family $(R=0.523$, $p<0.001$ ), and insulin levels and the Acidaminococcus genus $(R=0.539, p<0.001)$ in women without MetS.

\subsection{Diversity of the Gut Microbiota according to Gender in MetS Patients}

No significant differences in the richness and alpha-diversity parameters (Chao1, Simpson, and Shannon) were observed (Table S3, Supporting Information). Regarding beta-diversity, we detected significant differences between groups in the principal component analysis, based on unweighted (qualitative) and weighted (quantitative) UniFrac distance metrics (Figure S1, Supporting Information) analyzed by PERMANOVA ( $p=0.004$ and $p=0.006$, respectively). Moreover, we found significant differences in unweighted and weighted UniFrac distance metrics between NonMetS women and men (Figure S2, Supporting Information; PERMANOVA; $p=0.030$ and $p=0.001$, respectively). However, we found significant differences in unweighted UniFrac distance metrics between women and men with MetS (Figure S3, Supporting Information), but no statistically significant differences were found in weighted UniFrac distance metrics (PERMANOVA; $p=0.036$ and $p=0.437$, respectively).

\subsection{Differences in the Gut Microbiota between Genders in MetS: LEfSe Analysis}

In order to evaluate whether the dysbiosis of the gut microbiota in MetS differs by gender, we assessed the global differences of the gut microbiota between women and men with MetS. More- over, to discern whether these gender differences were specific in MetS, we compared these differences with those found between genders in the NonMetS groups.

We used LEfSe to compare the estimated phylotypes of the women and men in the MetS and NonMetS groups (Figure 1). The NonMetS men's gut microbiota was characterized by a preponderance of Firmicutes phylum and several genera from the Clostridiales order, such as Clostridium, Coprococcus, Dorea, Lachnospira, Roseburia, and Veillonella. By contrast, the MetS men's gut microbiota was characterized by a preponderance of genera such as Clostridium, SMB53, Coprococcus, Roseburia, and Faecalibacterium, but by a lower abundance of the Ruminococcus, Anaerotruncus, and Phascolarctobacterium genera.

On the other hand, the NonMetS women's gut microbiota was characterized by a preponderance of families and genera from the Bacteroidales order, such as Bacteroides, Barneciellaceae, Butyricimonas, Parabacteroides, and Rikenellaceae, whereas the MetS women's gut microbiota was characterized by a preponderance of the Cyanobacteria phylum and Parabacteroides genus, but by lower Prevotella genus.

\subsection{Differences in the Abundance of the Microbial Taxa between Genders in MetS: Univariate Analysis}

Further, we explored the MetS-specific differences in the abundance of bacterial taxa at genus level between women and men by one-way ANOVA, adjusting the FDR by the Benjamini and Hochberg method. Moreover, we also analyzed the differences between women and men in NonMetS control groups to discern whether the gender differences found were dependent or independent of MetS (Figure 2).

In fact, we found specific gender differences in MetS groups not present in the NonMetS control groups. We observed a higher abundance of the Collinsella, Alistipes, Anaerotruncus, and Phascolarctobacterium genera in MetS-W than in MetS-M $(p=0.003$, $q=0.021 ; p=0.014, q=0.088 ; p<0.001, q=0.001$; and $p=0.016, q=0.090$, respectively), whereas the abundance of Faecalibacterium and Prevotella genera was higher in MetS-M than in MetS-W $(p=0.002, q=0.015$ and $p<0.001, q=0.005$, respectively).

Moreover, we observed gender differences in MetS groups also present in NonMetS control groups-in other words, gender differences that were independent of MetS. We found higher levels of Ruminococcus (Lachnospiraceae family) and Bilophila genera in both MetS-W $(p<0.001, q<0.001$ and $p=0.002, q=0.017$, respectively) and non-MetS-W ( $p=0.009, q=0.032$ and $p<0.001$, $q=0.005$, respectively) than in their corresponding male groups. By contrast, we observed higher levels of Clostridium and SMB53 genera in MetS-M than in MetS-W $(p<0.001, q=0.002$ and $p<0.001, q=0.001$, respectively), and in non-MetS-M than in NonMetS-W (both, $p=0.002, q=0.011$ ).

\subsection{Bacterial Prevalence Differentially Associated to Genders in MetS}

We also analyzed the MetS-associated differences in bacterial genera prevalence between the genders using the $\chi^{2}$ test. 

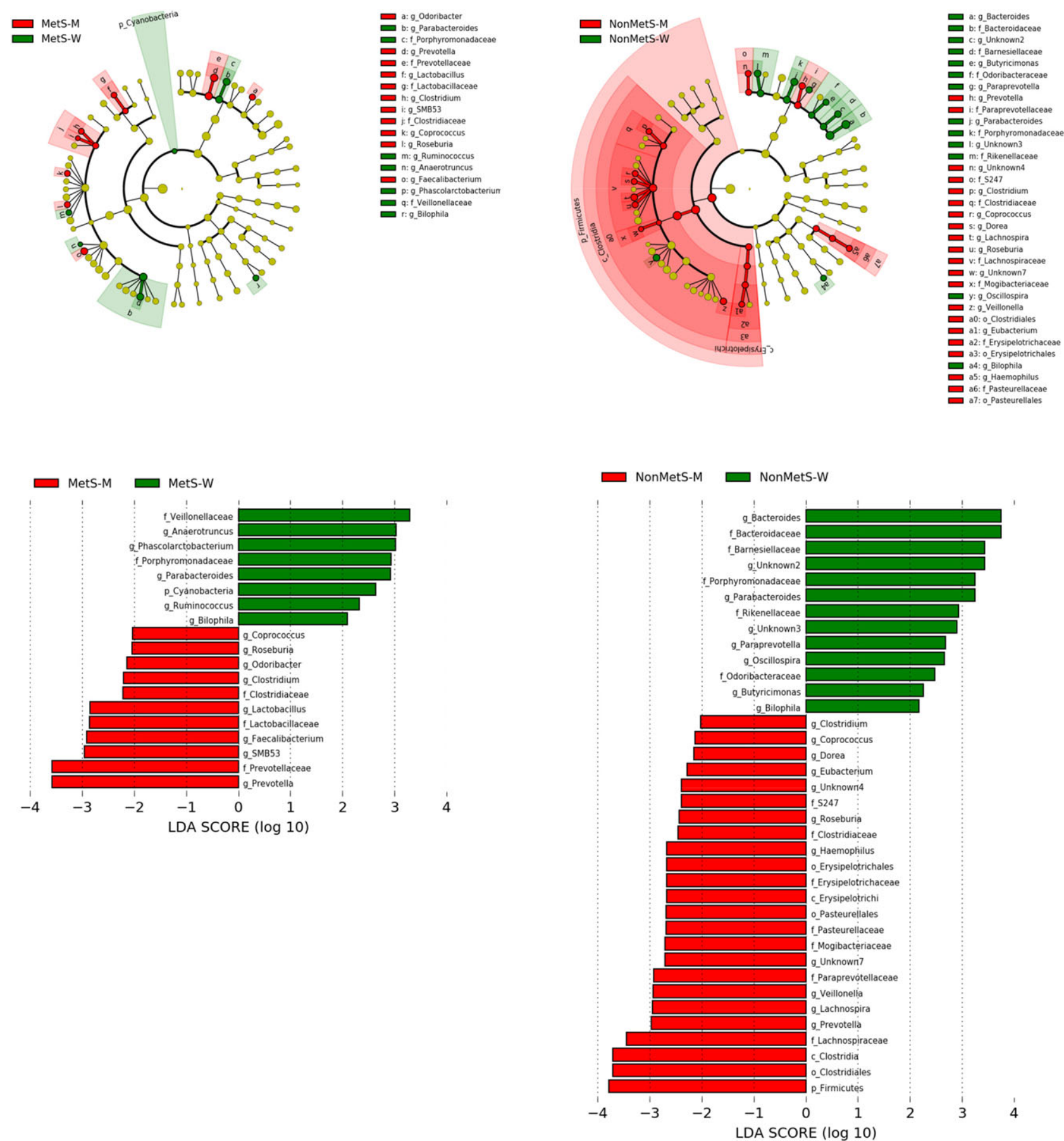

Figure 1. Linear discriminant analysis effect size. Cladogram representing the taxonomic hierarchical structure of the identified differences between genders using Linear discriminant analysis effect size. Each filled circle represents one phylotype. Red, bacterial taxa statistically overrepresented in men; green, bacterial taxa overrepresented in women. Phylum and class are indicated in their names on the cladogram and the order, family, or genus are given in the key.

Moreover, we analyzed the differences between women and men in the NonMetS groups to discern whether the gender differences found were dependent or independent of MetS (Table 2). A higher presence of Eggerthella $(67.1 \%$ vs $39.2 \%$; $\chi^{2}$ test $\left.p<0.001\right)$ and Acidaminococcus $\left(91.1 \%\right.$ vs $79.8 \% ; \chi^{2}$ test $p=0.042$ ) genera was observed in fecal samples from
MetS-W compared to MetS-M. Likewise, a higher presence of Slackia $\left(79.8 \%\right.$ vs $59.5 \% ; \chi^{2}$ test $\left.p=0.006\right)$, Odoribacter (100\% vs $93.7 \% ; \chi^{2}$ test $\left.p=0.023\right)$ genera, and an unknown genus from RF39 order $\left(59.5 \%\right.$ vs $39.2 \%$; $\chi^{2}$ test $p=0.011$ ) was observed in fecal samples from MetS-M compared to MetS-W. 


\begin{tabular}{|c|c|c|}
\hline \multicolumn{3}{|c|}{ MetS } \\
\hline \multirow{4}{*}{ BACTEROIDETES } & Alistipes & $P=0.014 ; Q=0.088$ \\
\hline & Odoribacter & $P=0.021 ; Q=0.105$ \\
\hline & Parabacteroides & $P=0.032 ; Q=0.138$ \\
\hline & Prevotella & $P<0.001 ; Q=0.005$ \\
\hline \multirow{7}{*}{ FIRMICUTES } & Anaerotruncus & $P<0.001 ; Q=0.001$ \\
\hline & Clostridium & $P<0.001 ; Q=0.002$ \\
\hline & Faecalibacterium & $P=0.002 ; Q=0.015$ \\
\hline & Phascolarctobacterium & $P=0.016 ; Q=0.090$ \\
\hline & Roseburia & $P=0.035 ; Q=0.140$ \\
\hline & Ruminococcus (L) & $P<0.001 ; Q<0.001$ \\
\hline & SMB53 & $P<0.001 ; Q=0.001$ \\
\hline \multirow{2}{*}{ ACTINOBACTERIA } & Bifidobacterium & $P=0.023 ; Q=0.105$ \\
\hline & Collinsella & $P=0.003 ; Q=0.021$ \\
\hline FUSOBACTERIA & Fusobacterium & $P=0.048 ; Q=0.179$ \\
\hline PROTEOBACTERIA & Bilophila & $P=0.002 ; Q=0.017$ \\
\hline
\end{tabular}

\begin{tabular}{|c|c|c|}
\hline \multicolumn{3}{|c|}{ NonMetS } \\
\hline \multirow{4}{*}{ BACTEROIDETES } & Alistipes & $P=0.186 ; Q=0.279$ \\
\hline & Odoribacter & $P=0.186 ; Q=0.279$ \\
\hline & Parabacteroides & $P<0.001 ; Q=0.003$ \\
\hline & Prevotella & $P=0.046 ; Q=0.105$ \\
\hline \multirow{7}{*}{ FIRMICUTES } & Anaerotruncus & $P=0.351 ; Q=0.447$ \\
\hline & Clostridium & $P=0.002 ; Q=0.011$ \\
\hline & Faecalibacterium & $P=0.122 ; Q=0.223$ \\
\hline & Phascolarctobacterium & $P=0.144 ; Q=0.245$ \\
\hline & Roseburia & $P=0.001 ; Q=0.006$ \\
\hline & Ruminococcus (L) & $P=0.009 ; Q=0.032$ \\
\hline & SMB53 & $P=0.002 ; Q=0.0$ \\
\hline \multirow{2}{*}{ ACTINOBACTERIA } & Bifidobacterium & $P=0.150 ; Q=0.248$ \\
\hline & Collinsella & $P=0.123 ; Q=0.223$ \\
\hline FUSOBACTERIA & Fusobacterium & $P=0.070 ; Q=0.151$ \\
\hline PROTEOBACTERIA & Bilophila & $P<0.001 ; Q=0.005$ \\
\hline
\end{tabular}

Figure 2. Differences in the gut microbiota composition according to gender in metabolic syndrome. Values represent mean of the genus, proportional in the four experimental groups (mean \pm SEM of the abundance are shown in Table S6, Supporting Information). Ruminococcus (L): Ruminococcus genus belonging to the Lachnospiraceae family. Statistical analysis was performed to find statistically significant differences in the abundance of each genus in the groups. $p$-Values: One-way ANOVA statistical analysis. $q$-Values: ANOVA p-values adjusted by the FDR using the Benjamini and Hochberg method.

\subsection{Differential Diet Effect on Gut Microbiota Composition}

In the next step, we explored whether gender determines a differential shaping of the gut microbiota from MetS patients by the consumption for 3 years of an MED or an LF diet. We observed higher levels of Desulfovibrio, Roseburia, and Holdemania genera in MetS-M than in MetS-W after the consumption for 3 years of the LF diet $(p=0.001, p=0.001$, and $p=0.023$ ), whereas no differences in the abundance of these bacterial genera between genders were observed after the consumption of the MED diet. By contrast, we found higher levels on an unknown genus from the Rikenellaceae family in MetS-M than in MetS-W after the consumption for 3 years of the MED diet $(p=0.018)$, whereas no differences were observed after the consumption of the LF diet (Figure 3).

Moreover, we also observed several gender-specific differences in the gut microbiota from MetS patients, regardless of the diet consumed. We observed a higher abundance of Suterella genus, and an unknown genera from Erysipelotrichaceae family and Enterobacteriaceae family in MetS-W than MetS-M after 3 years of dietary intervention $(p=0.001, p=0.001$, and $p=0.031$, respectively). By contrast, we observed a higher abundance of 
Table 2. Analysis of the bacterial genera presence according to metabolic syndrome between women and men. Values correspond to the number of patients in which the bacterial genus was detected; percentage is shown between brackets. The $\chi^{2}$ test was applied to establish differences in bacterial prevalence between the groups studied. Values: number of patients in which the genus was detected; ( $\%$ of patients in which the genus was detected); [Relative abundance of this genus in the group].

\begin{tabular}{|c|c|c|c|c|c|c|}
\hline & MetS-W $(N=79)$ & MetS-M $(N=79)$ & $\chi^{2}$ & NonMetS-W $(N=44)$ & NonMetS-M $(N=44)$ & $\chi^{2}$ \\
\hline \multirow[t]{2}{*}{ Eggerthella } & $53(67.09)$ & $31(39.24)$ & $<0.001$ & $24(54.55)$ & $20(45.45)$ & 0.394 \\
\hline & [0.00027] & {$[0.00014]$} & & [0.00052] & [0.00005] & \\
\hline \multirow[t]{2}{*}{ Slackia } & 47 (59.49) & $63(79.75)$ & 0.006 & $28(63.64)$ & $36(81.82)$ & 0.056 \\
\hline & {$[0.00072]$} & [0.00110] & & [0.00127] & [0.00213] & \\
\hline \multirow[t]{2}{*}{ Odoribacter } & $74(93.67)$ & $79(100)$ & 0.023 & $43(97.73)$ & $44(100)$ & 0.315 \\
\hline & {$[0.00318]$} & [0.00424] & & [0.00352] & [0.00283] & \\
\hline \multirow[t]{2}{*}{ Acidaminococcus } & $72(91.14)$ & $63(79.75)$ & 0.042 & $39(88.64)$ & $42(95.45)$ & 0.237 \\
\hline & {$[0.00137]$} & {$[0.00270]$} & & [0.00149] & {$[0.00028]$} & \\
\hline \multirow[t]{2}{*}{ Unknown genus ${ }^{\mathrm{a}}$} & $31(39.24)$ & 47 (59.49) & 0.011 & $21(47.73)$ & $27(61.36)$ & 0.199 \\
\hline & {$[0.00018]$} & {$[0.00016]$} & & {$[0.00018]$} & {$[0.00026]$} & \\
\hline \multirow[t]{2}{*}{ Anaerotruncus } & $67(84.81)$ & $60(75.95)$ & 0.161 & $38(86.36)$ & $30(68.18)$ & 0.042 \\
\hline & {$[0.00013]$} & {$[0.00005]$} & & {$[0.00008]$} & {$[0.00007]$} & \\
\hline \multirow[t]{2}{*}{ Catenibacterium } & $43(54.43)$ & $50(63.29)$ & 0.258 & $23(52.27)$ & $33(75.00)$ & 0.027 \\
\hline & [0.00196] & [0.00234] & & [0.00144] & [0.00340] & \\
\hline
\end{tabular}

a) Unknown genus (Unknown family; RF39 order).

Phascolarctobacterium genus and an unknown genera from the Clostridiales order and Coriobacteriaceae and S24-7 families in MetS-M than MetS-W after 3 years of dietary intervention $(p=0.044, p=0.005, p=0.021$, and $p<0.001$, respectively; Figure 4).

\section{Discussion}

This work provides evidence of a different gut microbiota composition in MetS, according to gender. Moreover, our study showed a differential shaping of the gut microbiota, according to the gender, in MetS patients, after the consumption for 3 years of an MED or an LF diet.

Previous data has indicated that the variability of the human population, related with factors such as gender, seems to affect gut microbiota composition. ${ }^{[16-18]}$ However, to the best of our knowledge, the gender differences in gut microbiota in the presence of metabolic disease have not been studied in humans. Nevertheless, studies in animal models have identified a direct interaction between gender differences in gut microbiota and the development of disease, ${ }^{[33]}$ suggesting that microbiota dysbiosis may be related with the dimorphism observed in the incidence of metabolic diseases. ${ }^{[34-36]}$ To explore gender differences in MetS, we compared women and men with the same pattern of MetS criteria, to avoid a factor of confusion since different combinations of MetS criteria may be associated with different gut dysbiosis and therefore may hinder the identification of gender differences. Moreover, we also took into account the fact that the baseline nutritional background was similar in all the participants, as this parameter can also modify the microbial composition of the intestine. ${ }^{[20]}$

The present work has shown that the microbiota of women with MetS is different to the microbiota of men with MetS. However, this difference is less pronounced in MetS than in NonMetS groups, on the basis of the linear discriminant analysis effect size. A lower bacterial diversity associated to disease, which has been previously described, ${ }^{[37]}$ may be responsible for this observation. In fact, we observed significant differences in quantitative and qualitative metrics (weighted and unweighted UniFrac distances) between genders in NonMetS groups, whereas only qualitative metrics (unweighted UniFrac distance) showed differences between genders in MetS groups. This also suggests that low-abundance taxa may be important in determining the differences in gut microbiota composition between genders in disease, on the basis that weighted UniFrac incorporates abundances to calculate distance, and the impact of low-abundance features is diminished, whereas unweighted UniFrac is more sensitive to differences in low-abundance features. ${ }^{[31]}$ However, the impact of differences in low-abundance features between genders requires further research.

Taking into account the potential role of gut microbiota in the development of MetS, we explored the specific gender differences between MetS groups that did not exist between NonMetS women and men groups. Thus, we observed differences in the abundance of bacterial genera previously related with the presence of T2DM, such as Collinsella, Faecalibacterium, and Prevotella, ${ }^{[38]}$ with insulin resistance, such as Phascolarctobacterium, ${ }^{[39]}$ and directly with MetS, such as Anaerotruncus. ${ }^{[40]}$ Considering the fact that the SCFA-producers Faecalibacterium and Prevotella ${ }^{[38]}$ are more abundant in men than in women, our results reinforce the idea that the loss of certain functions or features of the gut microbiota, such as the loss of the capacity to degrade carbohydrates to SCFA, is present in MetS, as previously described, ${ }^{[41]}$ but the current work suggests that this might occur due to the differential alteration of the gut microbiota in women and in men. Moreover, these alterations may also differentially affect the extent to which SCFA production might potentially be reduced in the gut of women and men. 

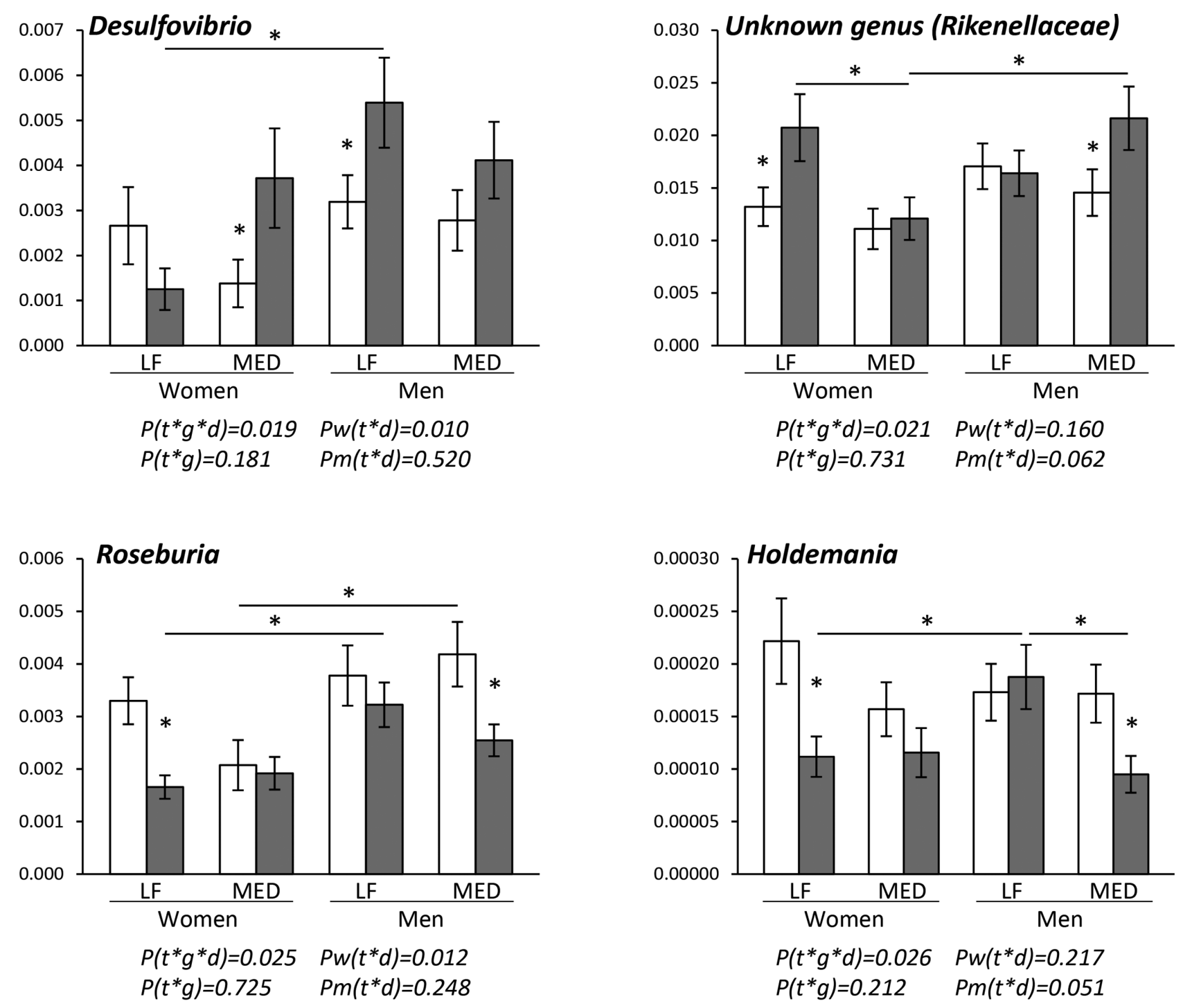

Baseline

Y3

Figure 3. Gut microbiota composition changes according to gender and diet. Values represent mean of the bacterial taxa abundance $\pm S E M$. LF, low-fat diet; MED, Mediterranean diet. The statistically significant differences between baseline and follow-up were tested by ANOVA for repeated measures, with time ( $\mathrm{t}$ ) as intrasubject factor, and diet $(\mathrm{d})$ and gender $(\mathrm{g})$ as the intersubject factors. $* p<0.05$ in the post hoc Bonferroni's multiple comparison test.

The fact that the same combinations of MetS criteria in women and men were associated to different alterations of the gut microbiota might help to explain the differences in the prevalence of MetS between genders, ${ }^{[3]}$ and the differences in the prevalence of each metabolic syndrome risk factor, which also differs by gender and country. ${ }^{[4,5]}$ Thus, it is plausible to think that the different dysbiosis or microbial imbalances in gut microbiota composition between men and women could be differentially favored or attained, which may therefore affect the incidence of the associated metabolic diseases between genders. In addition, given that each MetS combination may be associated to a different microbial dysbiosis, we identified a gender-dependent dysbiosis between women and men with the same combinations of MetS criteria.
Dietary strategies, which presumably act by modifying the microbiome, ${ }^{[20]}$ are of great interest in preventing and treating metabolic disorders. ${ }^{[19]}$ In fact, we have previously shown that the long-term consumption of both MED or LF diets partially restores the gut microbiota dysbiosis in obese people with the full characteristics of MetS (five criteria), whereas no significant modifications of gut microbiota were observed in NonMetS patients after the dietary intervention. ${ }^{[42]}$ Based on this, in the current work, we analyzed whether the long-term consumption of these diets induced different microbiota changes in men than in women with MetS. In fact, we found two different effects: a) bacterial taxa whose abundance changed differentially between genders as consequence of the dietary intervention and regardless of diet, and b) bacterial taxa whose abundance was differentially 
Sutterella

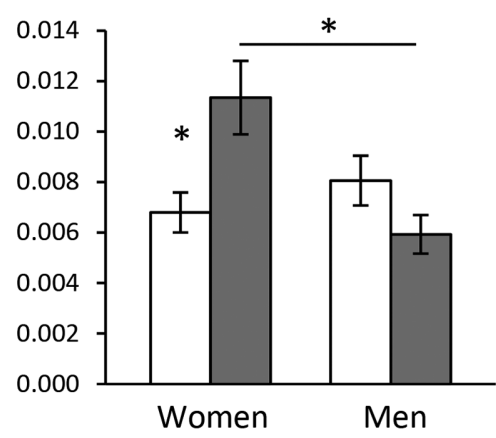

$P\left(t^{*} g^{*} d\right)=0.436 \quad P w\left(t^{*} d\right)=0.222$

$P\left(t^{*} g\right)=0.001 \quad P m\left(t^{*} d\right)=0.651$

Unknown genus

(Erysipelotrichaceae)

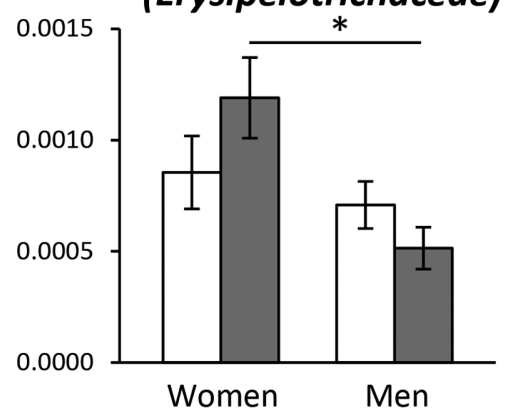

$P\left(t^{*} g^{*} d\right)=0.201 \quad P w\left(t^{*} d\right)=0.128$

$P\left(t^{*} g\right)=0.017 \quad P m\left(t^{*} d\right)=0.815$
Phascolarctobacterium

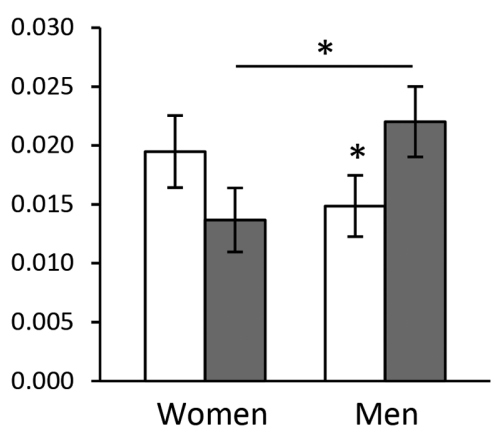

$P\left(t^{*} g^{*} d\right)=0.099 \quad P w\left(t^{*} d\right)=0.860$

$P\left(t^{*} g\right)=0.009 \quad P m\left(t^{*} d\right)=0.029$

Unknown genus

(Clostridiales)

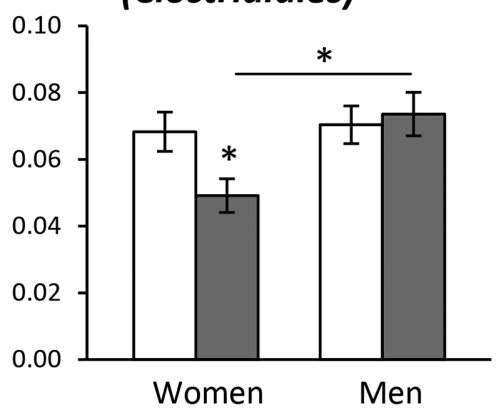

$P\left(t^{*} g^{*} d\right)=0.711 \quad P w\left(t^{*} d\right)=0.082$

$P\left(t^{*} g\right)=0.020 \quad P m\left(t^{*} d\right)=0.374$
Unknown genus

(Coriobacteriaceae)

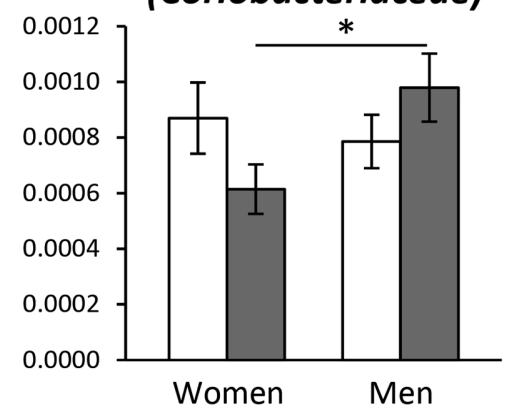

$P\left(t^{*} g^{*} d\right)=0.871 \quad P w\left(t^{*} d\right)=0.484$

$P\left(t^{*} g\right)=0.014 \quad P m\left(t^{*} d\right)=0.276$

Ruminococcus ( $R$ )

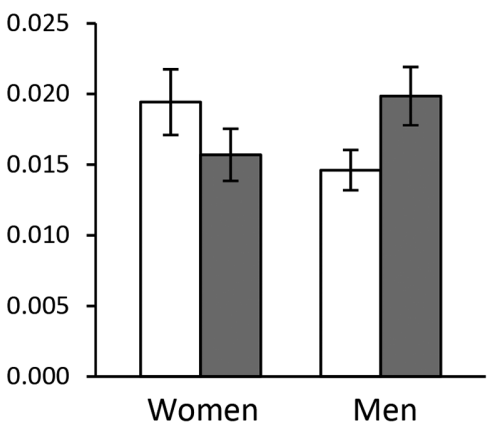

$P\left(t^{*} g^{*} d\right)=0.850 \quad P w\left(t^{*} d\right)=0.313$

$P\left(t^{*} g\right)=0.027 \quad P m\left(t^{*} d\right)=0.403$
Unknown genus

(Enterobacteriaceae)

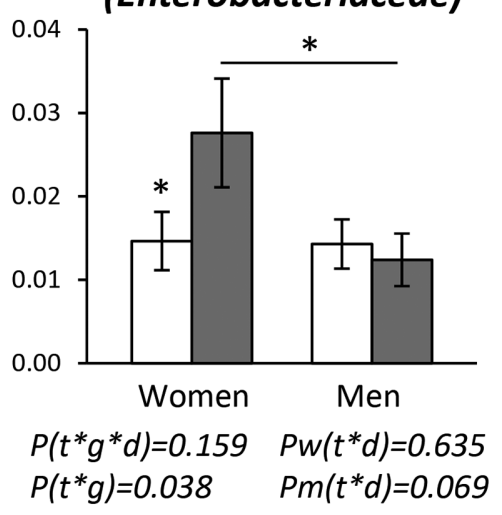

Unknown genus

(S24-7 family)

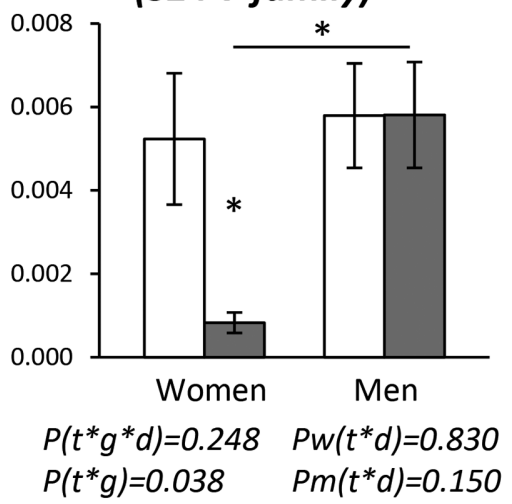

\section{Baseline}

Y3

Figure 4. Gut microbiota composition changes according to gender. Values represent mean of the bacterial taxa abundance \pm SEM. LF, low-fat diet; MED, Mediterranean diet. The statistically significant differences between baseline and follow-up were tested by ANOVA for repeated measures, with time ( $\mathrm{t}$ ) as intrasubject factor, and diet (d) and gender (g) as the intersubject factors. $* p<0.05$ in the post hoc Bonferroni's multiple comparison test. (R): Ruminococcus from Ruminococcaceae family. 
modified by the consumption of each diet, with a different effect in women than in men.

This latter is especially important, as our results suggest a new hypothesis based on the fact that women and men could differentially benefit from the consumption of the MED and LF diets. In fact, we observed a higher abundance of Roseburia, an SCFAproducer genus ${ }^{[4]}$ and Holdemania, a genus whose abundance is linked to impaired lipid and glucose metabolism, ${ }^{[44]}$ in men than in women after 3 years of the consumption of the LF diet. Moreover, we observed a higher abundance of Desulfovibrio in men than in women after 3 years of the consumption of this diet. The fact that this is a hydrogen sulfide-producing bacterial genus related with gastrointestinal disorders such as ulcerative colitis, Crohn's disease, and irritable bowel syndrome, ${ }^{[45]}$ suggests that the consumption of an LF diet has a detrimental effect in men with this pathology, whereas its consumption in women would be advisable. By contrast, the abundance of an unknown genus from the Rikenellaceae family, which has been shown to negatively correlate with MetS components, ${ }^{[46]}$ was higher in men than in women after 3 years of consumption of the MED diet. In addition, this bacterial genus was also higher in women who consumed the LF diet than in women who consumed the MED diet.

Our study has limitations. One limitation lies in the fact that although low-abundance taxa may be important in determining the differences in gut microbiota composition between genders in MetS, on the basis of beta-diversity metrics, the potential impact that differences in low-abundance features between genders may have on host physiology remains to be explored. Another limitation is that the 16S rRNA sequencing is suitable for microbiota analysis from phylum to genus levels, but is limited in its ability to identify bacterial species. Therefore, the analysis of the MetS-associated differences in bacterial taxa prevalence between the genders was performed at genus levels, and different bacterial species may be contributing to the prevalence of the genera.

In conclusion, our results suggest the potential involvement of the gut microbiota in the different incidence of metabolic diseases between genders. According to our results, which show the relationship between gut microbiota and metabolic disease, MetS-associated dysbiosis seems to be gender-dependent, and the alteration of the intestinal microbiota from normobiosis to dysbiosis may be different in women and men. Moreover, different diet-induced changes of gut microbiota by gender suggest that women and men could differentially benefit from the consumption of a specific diet, depending on their gender and disease.

\section{Supporting Information}

Supporting Information is available from the Wiley Online Library or from the author.

\section{Acknowledgements}

J.A.S.-M. and C.H. contributed equally to this work. J.A.S.-M., C.H., A.V.-R., J.F.A.-D., H.M.-A., A.L.-A., and J.L.-M. conducted the research; J.A.S.-M., C.H., A.V.-R., J.F.A.-D., H.M.-A., B.B.L., M.T.-S., and P.P.-M. analyzed the data or performed statistical analyses; J.A.S.-M., C.H., M.T.-S., F.P.J., and
A.C. wrote the paper; J.L.-M., F.P.-J., and A.C. designed the research; J.L.M., F.P.J., and A.C. had primary responsibility for the final content. The CIBEROBN is an initiative of the Instituto de Salud Carlos III, Madrid, Spain. The CORDIOPREV study is supported by the Fundación Patrimonio Comunal Olivarero, Junta de Andalucía (Consejería de Salud, Consejería de Agricultura y Pesca, Consejería de Innovación, Ciencia y Empresa), Diputaciones de Jaén y Córdoba, Centro de Excelencia en Investigación sobre Aceite de Oliva y Salud and Ministerio de Medio Ambiente, Medio Rural y Marino, Gobierno de España; Instituto de Salud Carlos III (CP14/00114 to A.C.). Ministerio de Economía y Competitividad (AGL2012/39615, PIE14/00005, and PIE 14/00031 to J.L.-M.; AGL201567896-P to J.L.-M. and A.C.; CP14/00114 to A C; PI13/00619 to F.P.-J.; PI16/01777 to F.P.-J. and P.P.-M.); Consejería de Innovación, Ciencia y Empresa, Proyectos de Investigación de Excelencia, Junta de Andalucía (CVI-7450 to J.L.-M.); and by the Fondo Europeo de Desarrollo Regional (FEDER). Antonio Camargo is supported by an ISCIII research contract (Programa Miguel-Servet CP14/00114). The authors would like to thank the Córdoba branch of the Biobank of the Sistema Sanitario Público de Andalucía (Andalusia, Spain) for providing the biological human samples. The authors also thank José Andrés Morales Martínez for technical assistance. The authors would also like to thank the EASP (Escuela Andaluza de Salud Pública), Granada, Spain.

\section{Conflict of Interest}

The authors declare no conflict of interest.

\section{Keywords}

dysbiosis, gut microbiota,metabolic diseases, obesity, sexual dimorphism

[1] P. M. Rao, D. M. Kelly, T. H. Jones, Nat. Rev. Endocrinol. 2013, 9, 479.

[2] S. M. Grundy, J. I. Cleeman, S. R. Daniels, K. A. Donato, R. H. Eckel, B. A. Franklin, D. J. Gordon, R. M. Krauss, P. J. Savage, S. C. Smith Jr., J. A. Spertus, F. Costa, Circulation 2005, 112, 2735.

[3] A. D. Pradhan, Clinical Chemistry 2014, 60, 44.

[4] R. B. Ervin, Natl. Health Stat. Rep. 2009, 5, 1.

[5] E. S. Ford, W. H. Giles, W. H. Dietz, JAMA, J. Am. Med. Assoc. 2002, 287, 356.

[6] F. Mauvais-Jarvis, Biol. Sex Diff. 2015, 6, 14.

[7] G. Enzi, M. Gasparo, P. R. Biondetti, D. Fiore, M. Semisa, F. Zurlo, Am. J. Clin. Nutr. 1986, 44, 739.

[8] T. Kelly, W. Yang, C. S. Chen, K. Reynolds, J. He, Int. J. Obes. 2008, 32, 1431.

[9] G. A. Rosito, J. M. Massaro, U. Hoffmann, F. L. Ruberg, A. A. Mahabadi, R. S. Vasan, C. J. O'Donnell, C. S. Fox, Circulation 2008, 117 , 605.

[10] S. Wild, G. Roglic, A. Green, R. Sicree, H. King, Diabetes Care 2004, 27, 1047

[11] V. Tremaroli, F. Backhed, Nature 2012, 489, 242.

[12] M. Bajzer, R. J. Seeley, Nature 2006, 444, 1009.

[13] I. Bondia-Pons, J. Maukonen, I. Mattila, A. Rissanen, M. Saarela, J. Kaprio, A. Hakkarainen, J. Lundbom, N. Lundbom, T. Hyotylainen, K. H. Pietilainen, M. Oresic, FASEB J. 2014.

[14] J. K. Nicholson, E. Holmes, J. Kinross, R. Burcelin, G. Gibson, W. Jia, S. Pettersson, Science 2012, 336, 1262. 
[15] M. J. Claesson, S. Cusack, O. O'Sullivan, R. Greene-Diniz, H. de Weerd, E. Flannery, J. R. Marchesi, D. Falush, T. Dinan, G. Fitzgerald, C. Stanton, D. van Sinderen, M. O'Connor, N. Harnedy, K. O'Connor, C. Henry, D. O'Mahony, A. P. Fitzgerald, F. Shanahan, C. Twomey, C. Hill, R. P. Ross, P. W. O'Toole, Proc. Natl. Acad. Sci. USA 2011, 1, 4586.

[16] C. Dominianni, R. Sinha, J. J. Goedert, Z. Pei, L. Yang, R. B. Hayes, J. Ahn, PLoS One 2015, 10, e0124599.

[17] C. Haro, O. A. Rangel-Zuniga, J. F. Alcala-Diaz, F. Gomez-Delgado, P. Perez-Martinez, J. Delgado-Lista, G. M. Quintana-Navarro, B. B. Landa, J. A. Navas-Cortes, M. Tena-Sempere, J. C. Clemente, J. Lopez-Miranda, F. Perez-Jimenez, A. Camargo, PLoS One 2016, 11, e0154090.

[18] S. Saraswati, R. Sitaraman, Front. Microbiol. 2015, 5, 764.

[19] C. J. Andersen, M. L. Fernandez, Rev. Endocr. Metab. Disord. 2013, 14, 241.

[20] A. R. Moschen, V. Wieser, H. Tilg, Gut Liver 2012, 6, 411.

[21] J. J. Faith, J. L. Guruge, M. Charbonneau, S. Subramanian, H. Seedorf, A. L. Goodman, J. C. Clemente, R. Knight, A. C. Heath, R. L. Leibel, M. Rosenbaum, J. I. Gordon, Science 2013, 341, 1237439.

[22] R. S. Kootte, E. Levin, J. Salojarvi, L. P. Smits, A. V. Hartstra, S. D. Udayappan, G. Hermes, K. E. Bouter, A. M. Koopen, J. J. Holst, F. K. Knop, E. E. Blaak, J. Zhao, H. Smidt, A. C. Harms, T. Hankemeijer, J. Bergman, H. A. Romijn, F. G. Schaap, S. W. M. Olde Damink, M. T. Ackermans, G. M. Dallinga-Thie, E. Zoetendal, W. M. de Vos, M. J. Serlie, E. S. G. Stroes, A. K. Groen, M. Nieuwdorp, Cell Metab. 2017, 26, 611. e6, e616.

[23] J. Delgado-Lista, P. Perez-Martinez, A. Garcia-Rios, J. F. Alcala-Diaz, A. I. Perez-Caballero, F. Gomez-Delgado, F. Fuentes, G. QuintanaNavarro, F. Lopez-Segura, A. M. Ortiz-Morales, N. Delgado-Casado, E. M. Yubero-Serrano, A. Camargo, C. Marin, F. Rodriguez-Cantalejo, P. Gomez-Luna, J. M. Ordovas, J. Lopez-Miranda, F. Perez-Jimenez, American Heart Journal 2016, 177, 42.

[24] Expert Panel on Detection, Evaluation, and Treatment of High Blood Cholesterol in Adults, A. Treatment of High Blood Cholesterol in, Executive Summary of The Third Report of The National Cholesterol Education Program (NCEP) Expert Panel on Detection, Evaluation, And Treatment of High Blood Cholesterol In Adults (Adult Treatment Panel III). JAMA, J. Am. Med. Assoc. 2001, 285, 2486.

[25] H. Schroder, M. Fito, R. Estruch, M. A. Martinez-Gonzalez, D. Corella, J. Salas-Salvado, R. Lamuela-Raventos, E. Ros, I. Salaverria, M. Fiol, J. Lapetra, E. Vinyoles, E. Gomez-Gracia, C. Lahoz, L. Serra-Majem, X. Pinto, V. Ruiz-Gutierrez, M. I. Covas, J. Nutr. 2011, 141, 1140.

[26] J. D. Fernandez-Ballart, J. L. Pinol, I. Zazpe, D. Corella, P. Carrasco, E. Toledo, M. Perez-Bauer, M. A. Martinez-Gonzalez, J. Salas-Salvado, J. M. Martin-Moreno, Br. J. Nutr. 2010, 103, 1808.

[27] A. Klindworth, E. Pruesse, T. Schweer, J. Peplies, C. Quast, M. Horn, F. O. Glockner, Nucleic Acids Res. 2013, 41, e1.

[28] J. G. Caporaso, J. Kuczynski, J. Stombaugh, K. Bittinger, F. D. Bush- man, E. K. Costello, N. Fierer, A. G. Pena, J. K. Goodrich, J. I. Gordon, G. A. Huttley, S. T. Kelley, D. Knights, J. E. Koenig, R. E. Ley, C. A. Lozupone, D. McDonald, B. D. Muegge, M. Pirrung, J. Reeder, J. R. Sevinsky, P. J. Turnbaugh, W. A. Walters, J. Widmann, T. Yatsunenko, J. Zaneveld, R. Knight, Nat. Methods. 2010, 7, 335.

[29] D. McDonald, M. N. Price, J. Goodrich, E. P. Nawrocki, T. Z. DeSantis, A. Probst, G. L. Andersen, R. Knight, P. Hugenholtz, ISME J. 2012, 6, 610.

[30] Ø. Hammer, Harper, D.A.T., P. D. Ryan, Palaeontol Electron. 2001, 4, 9.

[31] C. Lozupone, R. Knight, Appl. Environ. Microbiol. 2005, 71, 8228.

[32] N. Segata, J. Izard, L. Waldron, D. Gevers, L. Miropolsky, W. S. Garrett, C. Huttenhower, Genome Biol. 2011, 12, R60.

[33] J. G. Markle, D. N. Frank, S. Mortin-Toth, C. E. Robertson, L. M. Feazel, U. Rolle-Kampczyk, M. von Bergen, K. D. McCoy, A. J. Macpherson, J. S. Danska, Science 2013, 339, 1084.

[34] J. Arnlov, M. J. Pencina, S. Amin, B. H. Nam, E. J. Benjamin, J. M. Murabito, T. J. Wang, P. E. Knapp, R. B. D’Agostino Sr., S. Bhasin, R. S. Vasan, Ann. Intern. Med. 2006, 145, 176.

[35] P. Bhupathy, C. D. Haines, L. A. Leinwand, Womens Health. 2010, 6, 77.

[36] E. D. Luczak, L. A. Leinwand, Annu. Rev. Physiol. 2009, 71, 1.

[37] A. Mosca, M. Leclerc, J. P. Hugot, Front. Microbiol. 2016, 7, 455.

[38] M. Candela, E. Biagi, M. Soverini, C. Consolandi, S. Quercia, M. Severgnini, C. Peano, S. Turroni, S. Rampelli, P. Pozzilli, M. Pianesi, F. Fallucca, P. Brigidi, Br.J. Nutr. 2016, 116, 80.

[39] O. Polansky, Z. Sekelova, M. Faldynova, A. Sebkova, F. Sisak, I. Rychlik, Appl. Environ. Microbiol. 2015, 82, 1569.

[40] M. L. Zupancic, B. L. Cantarel, Z. Liu, E. F. Drabek, K. A. Ryan, S. Cirimotich, C. Jones, R. Knight, W. A. Walters, D. Knights, E. F. Mongodin, R. B. Horenstein, B. D. Mitchell, N. Steinle, S. Snitker, A. R. Shuldiner, C. M. Fraser, PLoS One 2012, 7, e43052.

[41] C. Haro, S. Garcia-Carpintero, J. F. Alcala-Diaz, F. Gomez-Delgado, J. Delgado-Lista, P. Perez-Martinez, O. A. Rangel Zuniga, G. M. Quintana-Navarro, B. B. Landa, J. C. Clemente, J. Lopez-Miranda, A. Camargo, F. Perez-Jimenez, The Journal of Nutritional Biochemistry 2016, 27, 27.

[42] C. Haro, S. Garcia-Carpintero, O. A. Rangel-Zuniga, J. F. Alcala-Diaz, B. B. Landa, J. C. Clemente, P. Perez-Martinez, J. Lopez-Miranda, F. Perez-Jimenez, A. Camargo, Mol. Nutr. Food Res. 2017, 61, 1700300.

[43] S. H. Duncan, R. I. Aminov, K. P. Scott, P. Louis, T. B. Stanton, H. J. Flint, Int. J. Syst. Evol. Microbiol. 2006, 56, 2437.

[44] K. Lippert, L. Kedenko, L. Antonielli, I. Kedenko, C. Gemeier, M. Leitner, A. Kautzky-Willer, B. Paulweber, E. Hackl, Benef. Microbes 2017, $8,545$.

[45] S. B. Singh, H. C. Lin, Microorganisms 2015, 3, 866.

[46] M. Y. Lim, H. J. You, H. S. Yoon, B. Kwon, J. Y. Lee, S. Lee, Y. M. Song, K. Lee, J. Sung, G. Ko, Gut 2017, 66, 1031. 